\title{
CIBERBULLYING HOMOFÓBICO EN EDUCACIÓN PRIMARIA: HACIA UNA ESCUELA LIBRE DE HOMOFOBIA
}

\author{
HOMOPHOBIC CYBERBULLYING IN PRIMARY EDUCATION: TOWARDS \\ A SCHOOL FREE OF HOMOPHOBIA
}
CYBERBULLYING HOMOFÓBICA NO ENSINO FUNDAMENTAL: RUMO A UMA ESCOLA LIVRE DE HOMOFOBIA

\author{
Begoña Sánchez Torrejón \\ Universidad de Cádiz, España \\ https://orcid.org/0000-0002-1242-4172 \\ begonia.sanchez@uca.es \\ Yarlenis Mestre Malfran \\ Universidad de La Habana, Cuba \\ http://orcid.org/0000-0003-1923-4811 \\ yarlenispsicodecuba@gmail.com
}

Recibido:12/03/2021 Revisado: 09/05/2021 Aceptado: 24/05/2021 Publicado: 20/07/2021

Resumen: En los últimos años ha aumentado de manera alarmante el ciberbullying, debido a la constante evolución de la tecnología, unido a la cada vez menor edad de acceso a los dispositivos tecnológicos. Además, el anonimato y la no percepción directa e inmediata del daño causado, convierten al ciberbullying homofóbico en un gran desafío que la escuela debe superar y dar respuesta. Necesitamos escuchar las experiencias y emociones vividas por el alumnado que a través de dispositivos tecnológicos han sido acosados por su orientación sexual en el ámbito escolar. El objetivo de este artículo es conocer las diversas consecuencias que provoca el ciberbullying homofóbico en el alumnado. Para ello se usa la metodología biográfica-narrativa para dar voz a un alumno de sexto de Educación Primaria que ha sufrido ciberbullying homofóbico por parte de algunos compañeros de su clase.

Palabras claves: Educación; Tecnologías; Ciberbullying; Homofobia; Inclusión.

\begin{abstract}
In recent years, cyberbullying has increased alarmingly, due to the constant evolution of technology, together with the ever-decreasing age of access to technological devices. In addition, anonymity, and the lack of direct and immediate perception of the damage caused, make homophobic cyberbullying a great challenge that schools must overcome and provide answers to. We need to listen to the experiences and emotions of students who have been harassed for their sexual orientation in the school setting through technological devices. The objective of this article is to learn about the several consequences caused by homophobic cyberbullying. For this purpose, the biographical narrative methodology is used to give a voice to a sixth-grade student of Primary Education who has suffered homophobic cyberbullying by some of his classmates.
\end{abstract}

Keywords: Education; Technologies; Cyberbullying; Homophobia; Inclusion.

Resumo: Nos últimos anos, o cyberbullying tem aumentado de forma alarmante devido à constante evolução da tecnologia aliada à idade cada vez menor de acesso aos dispositivos tecnológicos. Além disso, o anonimato e a não percepção direta e imediata dos danos causados fazem do cyberbullying homofóbico um grande desafio que a escola deve superar e responder. 
Precisamos ouvir as experiências e emoções vivenciadas por estudantes que foram assediados por meio de dispositivos tecnológicos em função da sua orientação sexual no âmbito escolar. $\mathrm{O}$ objetivo deste artigo é conhecer as diversas consequências causadas pelo cyberbullying homofóbico. Para isso, a metodologia biográfico-narrativa é utilizada para dar voz a um aluno do sexto ano do Ensino Fundamental que sofreu cyberbullying homofóbico por alguns colegas de aula.

Palavras-chave: Educação; Tecnologias; Cyberbullying; Homofobia; Inclusão.

Cómo citar este artículo: Sánchez-Torrejón, B., y (2021). Mestre, Y. Ciberbullying homofóbico en Educación Primaria: hacia una escuela libro de homofóbico. Hachetetepé. Revista científica en Educación y Comunicación, (23), 1-12. https://doi.org/10.25267/Hachetetepe.2021.i23.2202

\section{INTRODUCCIÓN}

Para abordar el problema del ciberbullying homofóbico en el ámbito escolar, es preciso situar a la escuela como uno de los espacios de reproducción de normas hegemónicas de género y sexualidad (Bento, 2011). Por medio del apelo a diversas tecnologías sociales, la escuela recrea el proyecto hegemónico de género que establece el binarismo como única posibilidad de existencia, la heterosexualidad obligatoria entre otros imperativos asentados en una lógica de destino natural. Sin embargo, como afirma Teresa de Lauretis (1987), género y sexualidad no corresponden únicamente a aquello que es totalizado en determinadas representaciones de lo femenino y lo masculino, sino aquello que excede las representaciones hegemónicas y las desestabiliza. Con base en este presupuesto también es posible pensar en la escuela como espacio en el que las narrativas hegemónicas pueden ser contestadas por medio de estrategias inclusivas.

Suscribimos entonces que las normas de género constituyen el punto de partida de múltiples violencias que alcanzan a las personas que se sitúan en los márgenes de la heterosexualidad obligatoria (Rich, 2012). Una de las formas de violencia que ha sido reconocida dentro del ámbito escolar es el ciberbullying homofóbico, una práctica que afecta al alumnado gay tanto en el ámbito escolar como en otras esferas vitales. Autores como Herrera et al. (2018), afirman que el ciberbullying es una práctica de intimidación, agresión continuada por medio de vías electrónicas como teléfonos móviles, redes sociales o WhatsApp. Alguno de los diversos efectos que produce este tipo de violencia son el bajo rendimiento académico, el absentismo escolar y la depresión entre otros.

Es posible reconocer que este tipo de acoso se ha visto facilitado por la masificación del uso de las tecnologías, las que hacen parte del cotidiano de vida de jóvenes y adolescentes en los centros escolares. Es por ello, que una de las condiciones que incide en el ciberbullying es la disponibilidad permanente del acceso a las tecnologías, lo que ocasiona que las víctimas puedan ser atacadas en cualquier lugar o momento, quedando los victimarios preservados por el anonimato que también facilitan dichas tecnologías. Otra de las singularidades de este tipo de violencia es la rápida viralización de los mensajes de odio, los que, al ser compartidos con una vasta audiencia, exponen a la víctima a un mayor sufrimiento (Mendoza, 2012).

Entre las modalidades de ciberbullying más mencionadas por algunos investigadores del tema se encuentra el insulto electrónico, hostigamiento, suplantación 
de la identidad de la víctima para revelar informaciones comprometedoras de esta, la exclusión y el ostracismo, la ciberpersecusión como una modalidad de comunicación reiterativa e insistente con finalidad de persecución (Mendoza, 2012; Giménez, 2015).

Sin dudas, esta constituye una problemática de especial relevancia e incidencia en el entorno educativo. Como postulan Carretero y Nolasco: "el reto de construir un sistema escolar inclusivo y diverso, donde lo diferente no sea valorado de manera negativa, tendría también una repercusión positiva en la disminución de las situaciones de acoso escolar" (2021, p.171). Precisamente una de las fuentes del ciberbullying es la homofobia, toda vez que ella forma parte de la diseminación de imaginarios heteronormativos de los que la escuela forma parte. En la siguiente sección profundizamos en las manifestaciones de la homofobia en el ámbito escolar en la etapa de Educación Primaria, lo que servirá de punto de partida para retomar seguidamente el ciberbullying homofóbico como una práctica específica dentro del universo de las violencias que afectan al alumnado gay. Al colocar este debate, resaltamos sobre todo la necesidad de diseñar estrategias de prevención de este fenómeno, como una prioridad de las agendas educativas en la construcción de una escuela inclusiva con todo el alumnado. Como señalan Cristancho y Niño: "En este sentido, para dar un tratamiento efectivo al problema es importante recurrir a estrategias de formación y prevención, que permitan atenuar dichas situaciones" (2020, p.112).

Entre las diversas razones por las cuales valoramos necesario el presente estudio, contemplamos el auge del ciberbullying en las escuelas españolas: "Las situaciones de bullying y ciberbullying son, tristemente, acontecimientos cada vez más presentes en la comunidad escolar, especialmente favorecidas, en el caso del ciberbullying, por el gran auge de las redes sociales" (Palacios et al.,2013,p.161), asimismo de escasas investigaciones sobre ciberbullying en la etapa de Educación Primaria, además una mayor representación en la investigación desde un punto de vista adultocéntrica: principalmente en perspectivas del profesorado en activo y en formación, encontrándose una laguna de estudios que de voz al propio alumnado. Por lo tanto, los objetivos planteados son: Conocer las diversas consecuencias que provoca el ciberbullying homofóbico en el alumnado. Descubrir las intervenciones educativas frente al ciberbullying. Saber las diversas emociones que sufren las víctimas de ciberbullying.

\subsection{Homofobia en la etapa de Educación Primaria}

Para afrontar el problema de la homofobia en el sistema educativo es preciso conocer exactamente de qué estamos hablando, en términos generales se trata de una forma de discriminación y exclusión que tiene algunas características muy específicas. La vivencia, en términos de Gómez (2005) de cualquiera forma específica de homofobia por parte del alumnado tiene importantes consecuencias a nivel psicológico y social: baja autoestima, autoexclusión, problemas familiares, síntomas psicosomáticos $\mathrm{o}$ físicos, insatisfacción, ansiedad y depresión, llegando en casos extremos al suicidio. En el presente artículo trataremos la homofobia ejercida hacia el alumnado gay.

En el ámbito educativo hay que recalcar que todas estas experiencias de acoso y exclusión derivan, según Takács (2006), en mayores tasas de fracaso y abandono escolar entre el alumnado no heterosexual, lo que conlleva un impacto negativo en la capacidad de estas personas para adaptarse adecuadamente en el paso de la escuela, al mundo laboral y para llegar a ser adultas con confianza en sí mismas. El entorno de iguales es consciente de esta situación y permite que suceda, a espaldas de las personas 
adultas del entorno que desconocen, o que, a su vez, minimizan la situación tomando la decisión de ignorarla, y de esta forma son colaboradores por omisión de actuación en estas acciones de acoso (Platero y Gómez, 2007).

El informe "Infancias vulnerables" elaborado por Ararteko para el Parlamento Vasco, en el año 2011 afirma que la diversidad de orientaciones afectivo-sexuales, así como la relativa a la identidad transexual y transgénero no están debidamente reconocidas y no gozan aun de un estatus de normalidad en nuestra sociedad. Esto genera que muchos niños y muchas niñas y adolescentes LGBTI $^{1}$ convivan a diario con situaciones de no aceptación de su realidad, e incluso de rechazo abierto en sus familias, entorno escolar o de ocio.

Algunos elementos específicos del bullying homofóbico frente a otras formas de acoso escolar son según Molinuero (2007), la invisibilización de la educación formal en el sistema educativo; el rechazo familiar o la falta de apoyo específico y explícito ante las sexualidades minoritarias; el contagio del estigma, no sólo para lesbianas, gais, transexuales y bisexuales sino para quienes les apoyan; y la normalización de la homofobia, que provoca una interiorización negativa del autoconcepto. Así, Borillo define la homofobia como "la hostilidad general, psicológica y social, respecto a aquellos y aquellas de quienes se supone que desean a individuos de su propio sexo tienen prácticas sexuales con ellos. Forma específica del sexismo" (2001, p. 36).

La homofobia puede presentarse en diversas formas apunta Borrillo (2001), bien sea conjuntamente o en alguna de sus variaciones. Existe una homofobia cognitiva que tiene que ver con pensar que la homosexualidad es antinatural, pecaminosa o tener cualquier otra ideología que la presenta como inferior o indeseable.

Otra tipología es la homofobia afectiva, en la que nos encontramos con sentimientos de rechazo ante la homosexualidad y los homosexuales. Agustín (2009), señala que la homofobia afectiva se puede manifestar ante el contacto con otras personas LGBT o ante la simple posibilidad del contacto con personas LGBTI. Hernáiz Landáez y Márquez Santander (2011) destacan que este tipo de homofobia se da ante el contacto físico con personas LGBTI, la simple visión de estas personas o la visión de muestras de afecto entre personas LGBTI.

Continuando con otro tipo de homofobia, la homofobia liberal, quizás más sutil y difícil de detectar, pero no por ello menos presente como puntualiza Borrillo (2001). Esta hace referencia a aquella que permite o no la expresión de la homosexualidad en el espacio privado, pero que en ningún caso acepta que se haga pública.

En relación con las consecuencias a corto y a largo plazo, otro aspecto de gran importancia son los insultos y las descalificaciones que se dan en los centros escolares en relación a la orientación sexual y al que anteriormente hacíamos alusión. El hecho de que estos insultos se den desde la Educación Primaria (Plummer ,2001), antes de que el alumnado haya desarrollado su orientación sexual tienen graves consecuencias ya que condiciona la construcción de la identidad sexual, no sólo de la persona agredida, sino también de los que oyen aquellos insultos. El entorno conoce que se está produciendo el acoso escolar, y no actúa para pararlo sino que se comporta más agresivamente tras observar un acto de agresión, produciéndose un contagio social que inhibe la ayuda e incluso fomenta la participación en actos intimidatorios (Avilés, 2002).

\footnotetext{
${ }^{1}$ Son las siglas que hacen referencia a las personas lesbianas, gais, bisexuales, transexuales, e intersexuales.
} 
Para finalizar, resulta interesante hacer referencia al estudio realizado por CollPlanas et al. (2009) sobre los niveles de homofobia existentes en el alumnado de Educación Primaria y el alumnado de Educación Secundaria. Los resultados encontrados vienen a señalar que el acoso por homofobia se da desde la etapa de Educación Primaria y no desde la etapa de Educación Secundaria como en numerosas ocasiones se afirma en los trabajos que abordan estas cuestiones.

\subsection{Estrategias de prevención frente al ciberbullying homofóbico en Educación Primaria}

Como hemos venido suscribiendo a lo largo de este trabajo, la homofobia ha sido reconocida como uno de los disparadores de acoso por vías virtuales, haciendo parte del ciberbullying, y al mismo tiempo dotando a este de especificidades y vivencias particulares. Este tipo de acoso se convierte en un mecanismo social de reforzamiento del sistema sexo-género, por medio de la persecución de lesbianas y gais principalmente (Barbero, 2017).

Así, apoyados en el uso de diferentes aplicaciones como WhatsApp, Telegram, correo electrónico, las personas practican las más diversas formas de hostilidad hacia miembros de la comunidad LGBTI. Se trata de la diseminación de mensajes de aversión hacia estas personas, tratándolas como enfermas, desequilibradas (Garaigordobil y Larrain, 2020). El foco de este tipo de acoso es tanto las performances de género de gais y lesbianas, como su identidad, deseos y prácticas sexuales, los que se ven sometidos a escrutinio y deslegitimación a través de canales de comunicación personales como los aplicativos que mencionamos. A su vez, este tipo de tecnologías asegura, en cierta medida, una cierta impunidad a los acosadores, quienes se ven amparados en la distancia física que facilitan las mismas.

La homofobia se expande así al entorno online, variando en sus modalidades de presentación y en la frecuencia pues la presencia de estas tecnologías en la vida cotidiana contribuye para que este tipo de acoso acontezca en cualquier momento y horario de la vida de las víctimas, perturbando su equilibrio psicológico y particularmente su rendimiento escolar. De acuerdo con algunos estudios, en el ciberbullying LGTBIfóbico "toman parte muchos/as más alumnos/as que observan y conocen la existencia de estas agresiones" (Benítez, 2016, p.17) por lo que suma un público espectador que, si bien no es protagonista, actúa como cómplice de estas agresiones, causando sufrimiento a las víctimas quienes se saben objeto de un escrutinio virtual colectivo.

Algunos estudios previos centrados en la prevalencia de este fenómeno en las escuelas de España aseguran que ciberbullying es más recurrente en chicos que en chicas (Giménez, 2015). También afirman que las elevadas cifras de este fenómeno "sugieren la existencia de un gravísimo problema de LGBTIfobia en nuestra comunidad educativa. Es por ello esencial educar al alumnado en la denuncia e invertir en la prevención específica del ciberacoso LGBTfóbico" (Benítez, 2016, p.21).

Coincidiendo con estas posturas, entendemos la necesidad de profundizar en el estudio del ciberbullying homofóbico, principalmente priorizando la escucha a víctimas que han sufrido este tipo de acoso. Por todo ello, consideramos necesario establecer estrategias de prevención frente al ciberbullying homofóbico en la etapa de Educación Primaria, como medidas necesarias en la construcción de una escuela inclusiva con la diversidad sexo-genérica. 
La importancia de estrategias educativas de prevención es fundamental para evitar el ciberbullying homofóbico, estas medidas deben ser tomadas desde todos los miembros que forman la comunidad educativa, realizando un trabajo conjunto. Con respecto al profesorado, es prioritario y clave que tenga una buena y actualizada formación "No poseer una buena formación específica en diversidad sexogenérica, junto con los estereotipos sexistas que posee el profesorado, representan dos grandes hándicaps en la inclusión de la diversidad sexo-genérica" (Sánchez Torrejón, 2020, p.265).

Además valoramos la necesidad de incluir en el currículum escolar de forma explícita la diversidad sexo-genérica, pues facilitaría los procesos de la inclusión educativa. Uno de ellos es lograr la sistematización de estos contenidos para que no sean tratados de forma excepcional y puntual, y trabajar de manera integradora la heterosexualidad y las sexualidades no normativas.

En el plano curricular sería conveniente plasmarlos en los proyectos y las programaciones educativas donde se vean reflejadas todas las opciones sexuales y en las normas de convivencia del centro y del aula que confluyen en la comisión de convivencia. Como medida de prevención general proponemos dentro del Plan de Convivencia del Centro; específicamente como complemento indispensable la elaboración de un decálogo anti acoso que forma parte del propio Plan. Esto nos ayudará en la prevención de casos futuros de ciberbullying homofóbico en el contexto educativo, facilitando así un ambiente de convivencia y participación libre de violencia.

\section{METODOLOGÍA}

La metodología es el procedimiento que guía nuestra acción a la hora de llevar a cabo todo el proceso de investigación, con la pretensión de poder dar respuesta a la intención del estudio. Sus principales características son la construcción de un diseño flexible, abierto a constantes replanteamientos, a través del establecimiento de un vínculo horizontal entre quien investiga y los colaboradores del estudio (González, 1997). Por ende, este tipo de investigación tiene entre sus escenarios privilegiados a la vida cotidiana, permitiéndonos un acercamiento a problemáticas del tipo del ciberbullying presente en el entorno escolar.

De acuerdo con Taylor y Bodgan (1986), una perspectiva metodológica cualitativa exige que quien investiga considere al escenario y a las personas en una perspectiva holística, en la que estos no son reducidos a variables, sino considerados como un todo. Por ello, optamos por una metodología más de corte cualitativo e interpretativo, con la intención de dar a comprender los hechos que son contados por nuestro informante a través de su propia voz.

\subsection{Método: Biografía-Narrativa}

A través de la metodología biográfica-narrativa pretendemos conocer y comprender el modo en que las personas informantes tienden a percibir sus relaciones y vivencias sobre una situación "Una consigna narrativa es plantear algún tipo de conflicto para que ocurra una sutil evolución en lo que queremos exponer" (Amar Rodríguez, 2016, p. 979).

La metodología biográfica-narrativa nos lleva hacia el bello ejercicio del diálogo libre con nuestro informante, abriendo un itinerario de múltiples realidades. Además nos ofrece, la posibilidad de acercarnos a una realidad compleja, como es, en este caso el 
ciberbullying homofóbico en edades tempranas. Concretamente el instrumento utilizado ha sido la entrevista semiestructurada, el tiempo aproximado de está, ha sido unos treinta minutos. Por todo ello, valoramos esta metodología como camino idóneo para reflejar una realidad educativa desde la propia mirada y el sentir de un alumno que nos lleva a leer la realidad educativa.

\subsection{Muestra utilizada}

Sobre el informante que ha hecho posible a través de sus vivencias y sus palabras esta investigación, siempre respetando escrupulosamente su anonimato, podemos decir que es un alumno de once años, de sexto de Educación Primaria de un colegio público de la provincia de Cádiz. El centro está ubicado en la ciudad de Jerez de la Frontera, concretamente en un barrio socioeconómico de clase media.

Debemos tener siempre presente las conductas éticas en el ejercicio de la investigación científica y en el uso del conocimiento producido, uno de los principios éticos a tener en cuenta es el consentimiento informado. Los requisitos específicos del consentimiento informado como enumera González-Ávila (2002), incluyen la información sobre los riesgos, la finalidad, los beneficios y las alternativas a la investigación, la comprensión del sujeto de esta información y de su propia situación, y la toma de una decisión libre sobre si es conveniente su participación.

No podemos obviar la responsabilidad de la persona investigadora en relación a los sujetos que participan en su investigación ésta debe comprometerse con la protección de los informantes, así como su derecho a la privacidad y a su dignidad (Sandín, 2003). Al ser un menor de edad nuestro informante, y respetando la ética de la investigación, solicitamos previamente a través de una reunión el consentimiento de la madre, el padre y del niño. El acuerdo consciente del menor requiere que la persona que investiga le proporcione información con un lenguaje claro y adecuado para su edad. Así, todas las personas implicadas firmaron el consentimiento informado, el cual protege la libertad de elección de una persona, y se respeta su autonomía cumpliendo con los requisitos de voluntariedad, información y capacidad (Del Río, 2010).

\section{RESULTADOS}

Pasamos a presentar los resultados, estos surgen a partir del proceso de diálogo de las diversas secuencias de la realidad subjetiva que dan a comprender la visión del mundo de nuestro informante, alumno de sexto de Educación Primaria. A través de su voz, procuramos acercarnos a la realidad de este alumno en su centro escolar. Estamos frente a un alumno que le ha gustado siempre ir a la escuela, es un niño muy entusiasta:

A mí siempre me ha gustado ir al colegio, porque me lo paso bien con mis amigos de siempre, jugando en el recreo, en la clase me gusta aprender cosas nuevas siempre, me gusta mucho leer, y se me da muy bien las matemáticas. También me encanta el atletismo, es lo que más me gusta de las clases de educación física.

Además, comenta que lo que más le gusta de la escuela es relacionarse con sus amigos y amigas, los cuales conserva desde la etapa de Educación infantil, y son muy importantes para él, ya que entre ellos mantienen una relación de apoyo, respeto y cordialidad. Su grupo, está compuesto tanto de niños como de niñas. Expone que se lleva bien con todo su grupo, pues siempre están juntos y se apoyan en todo. Para él, el colegio es muy importante, pues además de poder relacionarse con sus amigos y amigas, es un alumno con muchas inquietudes y motivación hacia el aprendizaje 
escolar. Le gusta participar activamente en la clase y en las diversas actividades del centro: "Siempre vamos juntos a todos lados, en clase, en el recreo, voy a todos los cumpleaños de mis amigos y en verano también nos vemos, vamos a la piscina de mi urbanización".

Nuestro informante comenta que ha sufrido por parte de algunos compañeros de clase ciberbullying: "Bueno... pues en el grupo del WhatsApp de clase me insultaron, se metían conmigo mucho y me sentí muy triste..."

El acoso a través del grupo de WhatsApp, se ha producido varias veces, la primera vez, el alumno no les comentó a sus padres los insultos y las burlas que estaba recibiendo, pues nos relata que se sentía muy perdido y asustado. En el grupo de WhatsApp de la clase, se realizaron insultos reiterativamente sobre su orientación sexual como "maricón" y "nenaza" además de risas y mofas sobre su manera de hablar. También, nos expresa el sentimiento de vulnerabilidad que ha experimentado ante la situación de acoso a través del WhatsApp. Este contexto de ciberbullying le ha inducido al aislamiento:

Me he sentido muy triste y no quería estar con nadie, ni con mis mejores amigos, ni siquiera con mi madre ni con mi padre, quería estar solo a mi aire, que me dejara en paz todo el mundo...

Además ha incidido negativamente en su rendimiento escolar: "Yo no quería ni estudiar, no podía, no tenía ganas de ir..."

Asimismo, ha incidido en la relación con sus familiares, pues nos comenta que tampoco quería hablar con sus propios padres. Incluso matiza que durante el episodio de ciberbullying la relación con sus padres se deterioró debido a continuas discusiones, alude que sentía mucha tristeza y se encontraba perdido. Además debido al hostigamiento y acoso continuo que ha sufrido a través del grupo de WhatsApp, esto le ha provocado varias secuelas a nivel psicológico como ansiedad y estrés. Incidiendo de manera muy negativa en su autoestima y la percepción de su orientación sexual, es significativo destacar que ha necesitado el apoyo profesional de un psicólogo para superar esta situación. Al igual que con sus amigos más íntimos de clase, tampoco quería hablar, aunque estos se preocupasen por él. Nuestro informante, en un principio no era capaz de verbalizar los episodios de ciberbullying. Nos comenta que cuando les comunicó a sus padres los acontecimientos, sintió alivio y apoyo:

Mis padres me han escuchado y ayudado mucho, aunque yo al principio no quería ni

hablar con ellos de lo que me pasó, pero menos mal que pude contárselo a ellos, me sentí mejor, y el psicólogo después también me ha ayudado mucho...

Con respecto a la actuación del centro educativo, los padres tras conocer los sucesos, contactaron con la tutora. Primeramente nuestro informante relató lo sucedido en una tutoría y además sus padres mantuvieron otra tutoría con el director del centro y la tutora:

Tuve una tutoría con mi profesora, donde le conté lo que había pasado en el grupo de WhatsApp, yo no quería porque tenía miedo y no quería hablar de lo que pasó, pero al final me vino bien, porque me ayudó...

Nuestro informante nos relata que en estas primeras reuniones, el centro tomo medidas con respecto a los compañeros de clase que habían llevado a cabo los insultos homofóbicos en el WhatsApp. A través de tutorías mantenidas con los niños agresores, los cuales pidieron perdón en público y se retractaron ante toda la clase sobre sus acciones. En esta línea, la docente ha trabajado en el aula el respeto a las diversas 
orientaciones sexuales. Estas medidas han provocado un cambio positivo: "Ahora ya estoy mejor, y no me da miedo ir al colegio y seguir aprendiendo con mis amigos de clase..."

\section{DISCUSIONES}

Es necesario, a continuación, expresar el sentido de la información intercambiada con nuestro informante para llegar a comprender los múltiples significados de los resultados. Este, proyecta una visión de una situación de ciberbullying homófobico, la cual le ha generado muchos miedos, e inseguridades.

Asimismo, le ha repercutido directamente en su relación con la escuela, pues anteriormente al episodio de ciberbullying, nuestro informante estaba muy motivado e ilusionado en el colegio. Durante un largo tiempo ha estado en silencio, sin poder expresar a nadie su dolorosa situación: "No se lo dije a nadie, ni a mis padres, ni a mi mejor amigo, porque me agobiaba mucho y tenía miedo..."

Observamos el efecto de aislamiento de la víctima al sufrir esta situación de acoso: "No quería ver, ni hablar por el móvil con mis amigos de siempre, no tenía ganas de nada..."

Esta situación de silencio, le ha provocado mucha rabia y deterioro de la relación con sus padres, la cual era muy cercana y positiva, hacia un aislamiento: "No quería estar con nadie, porque cuando mis padres me preguntaban que me pasaba, terminábamos peleando y yo me sentía todavía peor..."

El hecho de que toda la clase había visto en el grupo de WhatsApp los insultos, le creo mayor vulnerabilidad: "Yo cuando pensaba que toda la clase había visto como se habían reído de mí, no podía soportarlo, me daba mucha pena..."

Se detecta como la disponibilidad permanente del acceso a las tecnologías, ocasiona que las víctimas puedan ser atacadas en cualquier momento, incidiendo en su inestabilidad emocional, además de la rápida viralización de los mensajes que son compartidos y vistos por muchas personas, en este caso toda la clase.

Igualmente ha tenido un impacto perjudicial directo en su rendimiento escolar, ya que nos comenta que no podía concentrarse, ni tenía ganas de estudiar. Después de haber vivenciado esta situación de acoso, muestra un rechazo absoluto al espacio escolar: "No quería ir nunca más al colegio, nadie me puede obligar a ir a un sitio que no me gusta, porque lo pasó mal y no quiero..."

Se refleja a través de sus palabras, las repercusiones perjudiciales que ejerce el ciberbullying, en el rendimiento escolar de la víctima y en el absentismo.

Además se han producido cambios nocivos en su comportamiento, pues se ha mostrado más inseguro, miedoso y nervioso, llegando a tener pesadillas, problemas de insomnio y falta de apetito. Detectamos una incidencia negativa directa del ciberbullying homofóbico en la salud mental y física de quien la padece y un deterioro de su estado emocional.

La intervención del centro escolar frente a la situación de ciberbullying, se centró principalmente en mantener un dialogo tanto con nuestro informante y su familia como con los niños agresores. Las diversas intervenciones didácticas con la clase para favorecer la inclusión de la diversidad sexo-genérica en el aula han tenido un resultado positivo. 


\section{CONCLUSIONES}

Muchas son las reflexiones que surgen de las palabras y vivencias en la escuela de nuestro informante, que nos lleva a las conclusiones finales. Esta oportunidad de escuchar al alumnado y compartir su realidad escolar, a través de una interacción cercana nos recuerda que es necesario empezar a dibujar una escuela inclusiva con el alumnado gay. A tenor de lo expuesto, una de las conclusiones a la que llegamos es la necesidad de establecer en los centros escolares medidas preventivas ante el ciberbullying homofóbico, como herramienta para evitar esta situación.

En esta línea, es interesante tener en cuenta como futuras líneas de investigación la colaboración de los familiares con los centros educativos, el desarrollo y la implementación de protocolos de actuación que indiquen qué pasos hay que dar en casos de agresiones y de ciberbullying homofóbico (Platero y Gómez, 2007). En la elaboración de este tipo de protocolos se debe tener en cuenta que el profesorado necesita herramientas de mediación que vayan más allá de la mera amonestación. Además, valoramos que debemos considerar el incidente como una oportunidad para tratar la cuestión del respeto y la inclusión de la diversidad sexo-genérica en el centro. Pero, sobre todo, toda acción de respuesta a este tipo de agresiones a través de entornos tecnológicos, debe abordarse con el asesoramiento adecuado y teniendo en cuenta que las medidas adoptadas no perjudiquen a la persona que ha sufrido la agresión.

Las palabras de nuestro informante nos reflejan la urgente necesidad de la formación del profesorado, como medida clave y futura línea de investigación en la prevención e intervención frente al ciberbullying homofóbico. Ante la identificación de que un alumno está sufriendo este tipo de acoso por parte de sus compañeros y compañeras de clase, la intervención debe ser inmediata. Planteando la necesidad de trabajo en red de diferentes profesionales de la educación, la intervención social, psicológica y jurídico. Además, es necesario, fomentar intervenciones que no se centren únicamente en el agresor, sino que abarquen todos los aspectos psicosociales de las personas implicadas (Álvarez 2007).

Entre las limitaciones del presente estudio encontramos el tamaño de la muestra, hay que tener en cuenta que el tamaño de la muestra es demasiado pequeño, y por tanto es difícil encontrar generalizaciones significativas. Además, dentro de las limitaciones de la persona que investiga es necesario tener en cuenta los efectos longitudinales, es decir el tiempo disponible para investigar un problema y medir el cambio o la estabilidad en el tiempo (Price y Murnan ,2004).

A modo de conclusión, es necesario, por tanto, establecer una visión de la escuela que contemple, en un plano de igualdad, las distintas modalidades de la sexualidad humana y más en concreto, las diferentes orientaciones sexuales, contribuyendo a la normalización social de la diversidad sexo-genérica en el ámbito escolar. Por consiguiente esta investigación expone la necesidad de medidas integrales que actúen preventivamente para evitar el ciberbullying: "la escuela parece reacia a reconocer que la violencia homófoba es un problema colectivo, que debe ser prevenido interviniendo sobre las causas y no sobre las víctimas" (Dorais, 2001, p.70).

CONTRIBUCIÓN DE LOS AUTORES: Begoña Sánchez Torrejón (Conservación de datos, Análisis formal, investigación, visualización, metodología, supervisión, 
validación, redacción: borrador original ) y Yarlenis Mestre Malfran (Conceptualización, adquisición de fondos, administración de proyectos, recursos, software, redacción: revisión y edición)

FINANCIACIÓN Esta investigación no recibió ninguna financiación externa.

\section{REFERENCIAS BIBLIOGRÁFICAS}

Amar Rodríguez, V. (2016). Leer la vida. Una investigación desde la perspectiva narrativa. Revista Latinoamericana de Ciencias Sociales, Niñez y Juventud, 14 (2), 975-986.

Avilés, J.M. (2002). Bullying. Intimidación y maltrato entre alumnos. Steeseilas.

Barbero, M de S. (2017). Hacerse hombre en el aula: masculinidad, homofobia y acoso escolar. Cadernos Pagu. (50), c175014. https://doi.org/10.1590/18094449201700500014

Bento, B. (2011). Na escola se aprende que a diferença faz a diferença. Estudos Feministas, 19 (2), 549-559.

Benítez, E. (2016). Ciberbullyng LGBT-fóbico. Nuevas formas de intolerância. Grupo de Educación de COGAM.

Borrillo, D. (2001). Homofobia. Bellaterra.

Carretero, R., y Nolasco, A. (2021). Acoso escolar y diversidad. Relación del acoso escolar con la percepción de normalidad en víctimas y agresores. Revista de Educación, (392) , 155-175.

Coll-Planas, G, Bustamante, G., y Missé, M. (2009). Transitant per les fronteres del gènere. Estratègies, trajectòries i aportacions de joves trans, lesbianes i gais. Colección Estudis, 25. Departament d'Acció Social i Ciutadania. Secretaria de Joventut.

Cristancho, M. K., y Niño, J. A. (2020). Estrategia de formación docente para la intervención en casos de ciberbullying. Infometric@ - Serie Sociales Y Humanas, 3(1), 109-130.

Del Río, C. (2010). El consentimiento informado en menores y adolescentes: Contexto ético-legal y algunas cuestiones problemáticas. Información psicológica, (100), 60-67.

Dorais, M. (2001). Mort ou fif. VLB Éditeur.

Garaigordobil, M., y Larrain, E. (2020). Acoso y ciberacoso en adolescentes LGTB: Prevalencia y efectos en la salud mental. Comunicar, 28(62), 79-90. https://doi.org/10.3916/C62-2020-07

Giménez, A. G. (2015). Cyberbullying. Análisis de su incidencia entre estudiantes y percepciones del profesorado [Tesis doctoral]. Universidad de Murcia. https://bit.ly/36NixIH

Gómez, A.B. (2005). El impacto de la homofobia y el bullying anti gay en los/as jóvenes. En J. Genérelo y J.I. Pichardo (Coords), Homofobia en el sistema educativo (pp.93-105). Cogam.

González, F (1997). Epistemología cualitativa y subjetividad. Pueblo y Educación.

González-Ávila, M (2002). Aspectos éticos de la investigación cualitativa. Revista iberoamericana de educación, 29, 85-103.

Hernáiz Landáez, E., y Márquez Santander, D. (2011). Manual educativo para la diversidad. Fundación para los reflejos de Venezuela. 
Herrera, M., Romera, E., y Ortega-Ruiz, R. (2018) Bullying y cyberbullying en latinoamérica. Un estudio bibliométrico. Revista Mexicana de Investigación Educativa, 23(76), 125-155.

Institución del Ararteko (2011). Informe Infancias Vulnerables. Parlamento Vasco.

Lauretis, T. (1987). A tecnologia do gênero. University Press.

Molinuero, B. (2007) Actitudes ante la diversidad sexual de la población adolescente de Coslada (Madrid) y San Bartolomé de Tirajana (Gran Canaria). Ayuntamientos de Coslada y San Bartolomé de Tirajana. Federación Estatal de Lesbianas, Gays, Transexuales y Bisexuales.

Mendoza, E. (2012). Acoso cibernético o cyberbullying: Acoso con la tecnología electrónica. Pediatría de México, 14 (3), 133-146.

Palacios, V., Polo, Ma I., Felipe, E., León, B., y Fajardo Bullón, F. (2013). European Journal of Investigation in Health, Psychology and Education, 3(2), 161-170.

Platero, R. y Gómez, E. (2007). Herramientas para combatir el bullying homofóbico. Editorial Talasa.

Plummer, D. (2001). The Quest for Modern Manhood: Masculine Stereotypes, PeerCulture and the Social Significance of Homophobia. Journal of Adolescence, 24(1), 15-23.

Price, J.H., y Murnan, J. (2004). Research Limitations and the Necessity of Reporting Them. American Journal of Health Education, 35(2), 66-67.

Rich, A. (2012). Heterossexualidade compulsória e existência lésbica. Bagoas - Estudos gays: gêneros e sexualidades, 4(5), 18-44.

Sánchez Torrejón, B. (2021). La formación del profesorado de Educación Primaria en diversidad sexo-genérica. Revista Electrónica Interuniversitaria de Formación del Profesorado, 24(1), 253-266. https://doi.org/10.6018/reifop.393781

Sandín Esteban, M.P. (2003). Investigación cualitativa en educación: fundamentos y tradiciones. McGraw-Hill.

Takács, J. (2006). Social exclusion of young lesbian, gay, bisexual and transgenderpeople (LGBT) in Europe. ILGA Europe.

Taylor, S., y Bogdan, R. (1986). Introducción a los métodos cualitativos de investigación. La búsqueda de significados. Paidós. 